\title{
Endometrial Cancer Stem Cells: Are They a Possible Therapeutic Target?
}

\author{
Satoru Kyo
}

Published online: 11 December 2012

(C) Springer Science+Business Media New York 2012

\begin{abstract}
The dynamic regeneration of the human endometrium during the menstrual cycle is thought to be supported by a specialized cell population, probably residing in the basal layer, which continues to provide daughter cells with high proliferative potential. This cell population is assumed to have stem cell characteristics. Recent molecular studies have revealed the presence of putative epithelial and stromal stem cells in the endometrium. A cell population with stem cell characteristics also has been identified in malignant tumors and is known as cancer stem cells (CSC). Putative CSCs are prospectively isolated using methods based on either a surface marker or intracellular enzyme activity and then assessed by serial transplantation of sorted subpopulations of tumor cells into immunocompromised mice. Unlike many other tumor types, little information on the characteristics of CSCs in endometrial cancer is available. We and others have demonstrated that prospectively isolated $\mathrm{CD}_{133^{+}}$cells and/or side population cells in endometrial cancer are capable of initiating tumor formation in immunocompromised mice and recapitulating the phenotype of the original tumor and, therefore, are potential endometrial CSCs. This review summarizes key elements of our understanding of the normal endometrial stem cells and endometrial CSCs and further discusses a potential strategy to target endometrial CSCs in cancer therapy.
\end{abstract}

Keywords Endometrium · Endometrial cancer stem cells . Stem cells · Cancer stem cells (CSCs) - Endometrial cancer . Therapeutic target $\cdot$ Cancer therapy

S. Kyo $(\square)$

Department of Obstetrics and Gynecology, Kanazawa University

Graduate School of Medical Science, 13-1 Takaramachi,

Kanazawa, Ishikawa 920-8641, Japan

e-mail: satoruky@med.kanazawa-u.ac.jp

\section{Introduction}

The human endometrium is characterized by dynamic remodeling during a woman's reproductive years, undergoing more than 400 cycles of regeneration, differentiation, and shedding. The endometrium can completely regenerate even after surgical resection of almost all endometrial tissue, suggesting the presence of a specific cell population residing in the basal layer of the endometrium, rather than in the functionalis or myometrium, which rapidly proliferates and expands to regenerate the endometrium [1-3]. This population is thought to consist of endometrial stem cells responsible for the high regenerative capacity of the endometrium. Recent technological advances in the identification of adult stem cells in various organs have greatly aided in the search for endometrial stem cells, and evidence of the presence of stem cells has been reported [4]. Studying endometrial stem cells will greatly enhance our understanding of the mechanisms of endometrial regeneration as well as various endometrial disorders, especially those associated with abnormal bleeding or proliferation.

In addition to adult stem cells in normal tissues, the concept of cancer stem cells (CSCs) has been proposed in the past decade, and their existence has been identified in a variety of tumor types. CSCs, a subpopulation of malignant cells with stem cell properties, can give rise to a hierarchy of proliferative and progressively differentiating cells, which contribute to the phenotypic and behavioral cellular heterogeneity in a tumor. These stem-like cells are characterized by their ability to give rise to new tumors in immunocompromised mice, usually requiring small numbers of cells. However, little information is available regarding endometrial CSCs. In the present review, we first review current information on normal endometrial stem cells and endometrial CSCs and discuss the relationship between them. Next, we introduce the clinical impact of CSCs in tumor behavior, 
especially in endometrial cancer. We then outline emerging molecular manipulations to target CSCs for cancer therapy. Finally, we propose a CSC-based molecular target therapy combined with conventional chemotherapeutic agents for endometrial cancer.

\section{Are Endometrial Stem Cells Present?}

Several lines of experimental evidence have suggested the presence of endometrial stem cells. These are summarized in our recent review article [4]. Stem cells present in the endometrium will exhibit clonal expansion, generating genetically identical daughter cells. We sought to explore the possibility that endometrial glands clonally expand in each menstrual cycle [5]. Normal endometria from hysterectomy specimens were subjected to collagenase-based digestion, and then single human endometrial glands from each patient were purified and analyzed using a polymerase chain reaction-based assay of nonrandom $\mathrm{X}$ chromosome inactivation of an $\mathrm{X}$-linked androgen receptor gene. The genomic DNAs recovered from all the glands showed a single band pattern in the gel after digestion with a specific restriction enzyme, indicating that they are composed of uniform cells with paternally or maternally methylated inactive alleles, suggesting a monoclonal origin [5]. The clonality of endometrial glands was further examined by using a mouse that harbors a green fluorescent protein $(G F P)$ gene on either the maternal or the paternal X chromosome [6]. In these mice, cells with the transgene on the active $\mathrm{X}$ chromosome are fluorescent, whereas those with the transgene on the inactive $\mathrm{X}$ chromosome are not [7]. Therefore, these "green mice" are useful for analysis of the clonality of organs in vivo. We removed the uteri of green mice and counted a total of 100 endometrial glands in randomly selected fields under a fluorescence microscope and found that individual glands of this mouse consisted of fluorescent or nonfluorescent cells in similar ratios, indicating the monoclonal composition of glandular cells.

A well-characterized feature of adult stem cells in vitro is the ability of a single cell to produce a colony when seeded at a low density on a plastic dish. Using freshly isolated endometrium from hysterectomy specimens, Chan et al. found that $0.22 \%$ of epithelial fractions and $1.25 \%$ of stromal fractions formed individual colonies of $>50$ cells/ colony within 15 days at cloning density $\left(300-500 / \mathrm{cm}^{2}\right)$ [8]. These findings suggest that rare clonogenic epithelial and stromal cells are present in human endometrium, which has high proliferative and self-renewal potential, therefore providing evidence for the presence of endometrial epithelial and stromal stem cells.

Stem cell subpopulations have been identified in many mammals, including humans, following treatment of cell populations with the fluorescent dye Hoechst 33342 [the so-called "side-population" (SP) cells] [9]. This identification is based on the concept that adult stem cell subpopulations have the ability to more rapidly efflux Hoechst dye than other cell populations, thereby producing a characteristic SP population that can be identified using fluorescenceactivated cell sorting analysis. Recent evidence suggests that the SP phenotype is associated with high-level expression of the ATP-binding cassette transporter protein ABCG2/Bcrp1 [10]. Kato et al. first isolated and analyzed SP cells in human endometrial tissue samples [11]. The proportion of SP cells varies widely $(0-5.11 \%)$ among samples, and most SPs are present in the $\mathrm{CD}^{-} \mathrm{CD}^{-} 3^{-}$fraction $\left(160^{\mathrm{CD} 9-, \mathrm{CD} 13-}\right.$ cells/200 SP cells). Morphologically, SP cells are small and round. When SP cells are maintained in DMEM containing $100 \mathrm{ng} \mathrm{ml}^{-1}$ interleukin (IL)-6, $10 \mathrm{ngml}^{-1}$ thrombopoietin, $100 \mathrm{ngml}^{-1}$ stem cell factor, and $10 \%$ fetal calf serum, feeder cells slowly proliferate and gradually form colonies, which can be successfully maintained for $>9$ months. In contrast, non-SP cells become flat in shape, show limited proliferation, and finally become senescent within 3 months, suggesting that a long-term repopulating phenotype is specific to SP cells.

The differentiation capacity of putative endometrial stem cells has been confirmed in several studies. Gargett et al. showed that clonally derived epithelial cells can form spher-

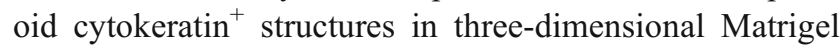
cultures, especially when cultured with stromal feeder cells [12]. Kato et al. reported that epithelial SP cells maintained in Matrigel cultures gradually aggregate after 4 months and finally form a gland-like structure that expresses CD9 and E-cadherin after 5 months [11]. In contrast, stromal SP cells do not form gland-like structures and do not express CD9 and E-cadherin, although they do express CD13 and vimentin.

Several groups have attempted in vivo transplantation of potential endometrial stem cells into immunocompromised mice. Masuda et al. transplanted endometrial SP cells (a mixture of epithelial and stromal SP cells) under the kidney capsule of ovariectomized severely immunodeficient NOD/ Shi-scid IL2Rgamma (null) (NOG) mice treated with estradiol. SP cells, but not non-SP cells, generated a cystic mass with delineated glandular and stromal structures at the site of transplantation [13]. Histological analyses of the transplantation sites revealed the presence of vascular components other than glandular and stromal components that were composed of $\mathrm{CD} 31^{+}$cells surrounded by $\alpha$-smooth muscle actin $(\alpha-\mathrm{SMA})^{+}$cells, suggesting that SP cells have the potential to differentiate into vessels. Cervello et al. implanted SP or non-SP cells into NOD-SCID mice and found that these cells from the epithelium do not give rise to any structures [14]. In contrast, SP cells from stromal fractions form macroscopic structures in the subcutaneous 
fat at the injection site. Histological analysis revealed that the structures are similar to the endometrial glands and stroma. This finding shows that stromal SP cells have the ability to form gland-like tissue in vivo. However, the possibility of contamination of stromal SP cells with small epithelial fractions cannot be denied, and further, careful, confirmative experiments are required.

Taken together, the emerging evidence suggests the presence of endometrial stem cells. Both epithelial and stromal stem cells are likely present. However, their origins are unknown, leaving several question unresolved; the possibility that more primitive common endometrial stem cells are present that can generate both epithelial and stromal stem cells or that stromal stem cells are a common source of epithelial and stromal cells.

\section{Concept of CSCs}

A small subpopulation of cells in malignant tumors was recently reported to have a high proliferative potential and contribute to tumorigenesis, proposing the concept of CSCs $[4,15]$. CSCs refer to a subset of tumor cells with the ability to self-renew and generate the diverse cells that comprise the tumor. These cells have been termed CSCs to reflect their "stem-like" properties and ability to continually sustain tumorigenesis. In CSC theory, a tumor shows a hierarchy analogous to the normal tissue hierarchy, with a restricted pool of cells showing self-renewal and differentiation potential. Differentiated tumor cells form the bulk of the tumor mass but are unable to self-renew. The "gold standard" for evaluating the presence of CSCs is serial transplantation of sorted subpopulations of tumor cells into immunocompromised mice. More simply, the activity of CSCs may be confirmed by a clonogenicity assay called a sphere-forming assay, in which colonies (e.g., mammospheres, neurospheres) originated from CSCs are formed on plastic dishes at clonal density in nonadherent culture or in Matrigel. However, colony-forming activity in such conditions may represent a readout of progenitor cell activity, rather than stem cell activity.

The existence of CSCs was first reported in acute myeloid leukemia (AML) $[16,17]$. Thereafter, evidence for the existence of CSCs has been demonstrated in a variety of solid tumors (Table 1) [18-30, 31•, 32•, 33•, 34-39]. Many of these studies have used the surface expression of CD133, a five-transmembrane glycoprotein, as a marker for the isolation of CSCs.

\section{Endometrial CSCs}

Very few studies regarding endometrial CSCs have been reported, most of which are introduced in our recent review article [4]. Hubbard et al. [40] examined the clonogenic activity of single endometrial carcinoma cells and revealed that most samples ( 28 of 31 ) form colonies with a cloning efficiency of $0.24 \%$, indicating that rare cells have the potential to initiate clones of daughter cells. As few as 10,000 cells established tumors in immunocompromised mice in 4 of 8 samples and formed an endometrioid-type adenocarcinoma similar to the parent tumor, recapitulating the expression of cytokeratin, vimentin, estrogen receptor $\alpha$, and progesterone receptor seen in parent tumors. Serial transplantation of cells from transplanted tumors resulted in development of additional tumors for up to five passages. These data suggest that the transplanted endometrial carcinoma cells contained a small number of CSCs that were capable of recapitulating the original tumor and differentiating in vivo.

We and another group recently showed that CD133 can be used as a marker of CSCs in endometrial cancer [4, 31•, 32•]. Our flow cytometric analysis using endometrial cancer cell lines revealed that sorted $\mathrm{CD} 133^{+}$cells show significantly
Table 1 Cancer stem cell markers in various tumors

ALDH, dehydrogenase; ESA, epithelial specific antigen; ABCG5, ATP-binding cassette sub-family G member 5

\begin{tabular}{|c|c|c|c|c|c|}
\hline Tumor type & CSC marker & References & Tumor type & CSC marker & References \\
\hline Brain & $\mathrm{CD}_{133^{+}}$ & [19] & Pancreas & {$\left[\begin{array}{l}\mathrm{CD} 133^{+} \\
\mathrm{CD}^{+} 4^{+} / 24^{+} / \mathrm{ESA}\end{array}\right.$} & $\begin{array}{l}{[28]} \\
{[29]}\end{array}$ \\
\hline Head and neck & $\mathrm{CD}_{4} 4^{+}$ & [20] & Colon & $\mathrm{CD}_{133^{+}}$ & {$[30]$} \\
\hline Breast & {$\left[\begin{array}{l}\mathrm{ALDH}^{+} \\
\mathrm{CD}^{+} 4^{+} / \mathrm{CD} 24^{-}\end{array}\right.$} & $\begin{array}{l}{[21]} \\
{[22]}\end{array}$ & Endometrium & {$\left[\begin{array}{l}\mathrm{CD} 133^{+} \\
\text {Side population }\end{array}\right.$} & $\begin{array}{l}{[32 \bullet]} \\
{[34]}\end{array}$ \\
\hline Lung & {$\left[\begin{array}{l}\mathrm{CD} 133^{+} \\
\mathrm{ALDH}^{+}\end{array}\right.$} & $\begin{array}{l}{[23]} \\
{[24]}\end{array}$ & Ovary & {$\left[\begin{array}{l}\mathrm{CD} 133^{+} \\
\mathrm{ALDH}^{+}\end{array}\right.$} & $\begin{array}{l}{[35]} \\
{[36]}\end{array}$ \\
\hline Liver & {$\left[\begin{array}{l}\mathrm{CD} 133^{+} \\
\mathrm{CD} 90^{+}\end{array}\right.$} & $\begin{array}{l}{[25]} \\
{[26]}\end{array}$ & Prostate & {$\left[\begin{array}{l}\mathrm{CD} 133^{+} \\
\mathrm{CD}^{+} 4^{+}\end{array}\right.$} & $\begin{array}{l}{[37]} \\
{[38]}\end{array}$ \\
\hline Stomach & $\mathrm{CD} 44^{+}$ & {$[27]$} & Melanoma & {$\left[\begin{array}{l}\mathrm{CD} 133^{+} \\
\mathrm{ABCG}^{+}\end{array}\right.$} & $\begin{array}{l}{[39]} \\
{[40]}\end{array}$ \\
\hline
\end{tabular}


increased colony-forming ability in vitro and tumorigenicity in nude or NOD/SCID mice than $\mathrm{CD} 133^{-}$cells, and furthermore, that $\mathrm{CD} 133^{+}$cells generate both $\mathrm{CD} 133^{+}$and $\mathrm{CD} 133^{-}$ cells, whereas $\mathrm{CD} 133^{-}$cells fail to do [31•]. Moreover, these $\mathrm{CD} 133^{+}$cells, unlike CD133 ${ }^{-}$cells, are apparently resistant to cisplatin- or paclitaxel-induced cytotoxicity. Rutella et al. examined primary tumor samples from patients with endometrioid-type endometrial cancer [32•] and observed a variable degree of CD133 immunoreactivity ranging from 1$60 \%$. Isolated $\mathrm{CD} 133^{+}$cells from tumor samples exhibit more aggressive proliferation in vitro and a greater cloning efficiency in single-cell colony assays compared with $\mathrm{CD} 133^{-}$tumor cells. When serially transplanted into NOD/SCID mice, $\mathrm{CD}_{133^{+}}$cells can initiate tumor formation and recapitulate the phenotype of the original tumor.

SP cells are present in various organs, including the endometrium, are known to exhibit stem characteristics, and have been identified as a cancer stem-like cell subpopulation in various cancers. Kato et al. isolated and characterized SP cells within a human endometrial cancer cell population [33•]. These SP cells showed long-term proliferative capacity in culture, self-renewal capacity in vitro, and enhanced tumorigenicity, compared with non-SP cells. In nude mice, these SP cells form large, invasive tumors that are composed of both tumor cells and stromal-like cells with an enriched extracellular matrix; the stromal-like cells contained human DNA, confirming that they were derived from the inoculated cells. Moreover, these SP cells differentiated into $\alpha$-SMA expressing cells in Matrigel. These findings demonstrate that these SP cells have cancer stemlike cell features and the potential to differentiate into cells of the mesenchymal cell lineage.

\section{Impact of CSCs in Tumor Behavior}

A high proportion of stem cells is generally believed to signify a worse prognosis. We investigated the clinical impact of CD133 expression in endometrial cancer [31•]. The expression of CD133 was immunohistochemically analyzed in 62 surgical specimens of endometrioid-type endometrial cancer. Twentysix of the 62 cancers $(41.9 \%)$ showed moderate or strong expression of CD133. There was no significant correlation between CD133 expression and any of the following: patient age, International Federation of Gynecology and Obstetrics (FIGO) stage, lymph node metastasis, depth of myometrial invasion, pathological grade, menopause, or body mass index. We next examined the prognostic impact of CD133 expression. The overall survival (OS) for the high-expression group was $69.2 \%$, and the OS for the low-expression group was $91.7 \%(p=0.023)$. The factor influencing the OS rate in univariate analysis was positive CD133 expression. Other factors that negatively influenced the OS rate in univariate analysis were the FIGO stage and histological grade. When these statistically significant variables from the univariate analyses were included in a Cox proportional hazard analysis, CD133 expression (hazard ratio, 3.9; $p=0.045$ ) and the FIGO stage (hazard ratio, 3.94; $p=0.042$ ) were identified as independent predictive factors of patient survival.

A similar clinical impact of CD133 expression on patient prognosis has been demonstrated in other tumor types, including brain [41, 42], colon [43], and pancreas tumors [44]. Other CSC markers have a similar clinical impact. High expression of aldehyde dehydrogenase (ALDH) is associated with poor prognosis in a number of tumor types, including lung [23], head and neck squamous cell carcinoma [45], pancreas [46], prostate [47], AML [48, 49], and breast [50-52]. The presence of $\mathrm{ALDH}^{+} \mathrm{CD} 133^{+}$cells in debulked primary tumor specimens correlates with poor patient survival [20]. Thus, patients with tumors that express high levels of molecules associated with CSCs are likely to have a poorer prognosis than those with tumors that express low levels of these markers.

One current question is why the presence of CSCs affects patient prognosis. Emerging evidence suggests that CSCs are involved in tumor invasion and metastasis, and poorer prognosis is probably seen in tumors expressing high levels of CSC markers. In our preliminary data, $\mathrm{CD} 133^{+}$endometrial cancer cells express higher levels of membrane-type 1 matrix metalloproteinase, causing increased invasive capacity (unpublished data). Expression of the CXC-chemokine receptor 4 (CXCR4) by tumor cells plays a critical role in cell metastasis [53]. Most CSCs express CXCR4 and migrate along a gradient of the CXCR4 ligand CXCL12, which is produced in hematopoietic niches, to facilitate metastasis. In endometrial cancer, CXCR4 is highly expressed by tumors, whereas the ligand CXCL12 is expressed by normal tissues [54]. In breast cancer, blocking CXCR4 expression in CSCs with antibodies or sh-RNA successfully inhibits the invasive behavior of tumor cells [55]. In the invasive front of pancreatic tumors, a distinct subpopulation of $\mathrm{CD}_{133^{+}} \mathrm{CXCR}^{+} \mathrm{CSC}$ was identified that determines the metastatic phenotype of the individual tumor. Their strong metastatic activity is primarily mediated by CXCR4, and the ligand CXCL12 is the most potent inducer of migration of $\mathrm{CD}_{133^{+}} \mathrm{CSCs}$ [28]. Continuous pharmacological inhibition of the CXCR4 receptor by AMD3100, a small-molecule inhibitor of CXCR4, significantly reduces tumor metastasis [28]. $\mathrm{ALDH}^{+} \mathrm{CSCs}$ among breast cancer cells showed a 413-gene expression profile that included genes known to play a role in stem cell function, as well as genes, such as CXCR1/IL-8 that were not previously known to play such a role. Recombinant IL8 treatment increases mammosphere formation and the $\mathrm{ALDH}^{+}$population. $\mathrm{ALDH}^{+} \mathrm{CSCs}$ show significantly increased invasive ability in response to IL-8, whereas the 
invasive ability of $\mathrm{ALDH}^{-}$cancer cells is not affected by IL8 treatment, indicating the importance of the CXCR1/IL8 axis in the invasive phenotypes of CSCs in breast cancer [56]. Thus, a subpopulation of CSCs is responsible for the invasive and metastatic phenotype of tumors, usually involving the chemokine ligand/receptor axis.

\section{Where do Endometrial CSCs Originate?}

One simple question is where endometrial CSCs arise. Researchers may first consider endometrial epithelial stem cells as the source of endometrial CSCs. This idea is easy to imagine, because normal stem cells have a self-renewal mechanism in place that allows for their longevity, including common signaling pathways such as the Wnt signaling pathway [57]. A growing tumor must contain self-renewing CSCs. As normal stem cells inherently possess this capacity, the path to a functional CSC may therefore be shorter than that for a differentiated somatic cell, which may have to acquire specific mutations to achieve the same self-renewing properties. Normal stem cells also clearly possess cancer-prone properties. Normal endometrial epithelial stem cells usually remain in a gland (probably in the basal layer) for much longer than differentiated glandular cells, which are lost by shedding in each menstrual period, offering a semipermanent target population for transformation. Therefore, the opportunity for normal stem cells to acquire transforming mutations may increase greatly.

Genetic alterations that occur in normal stem cells must be consistent and passed onto daughter cells through subsequent clonal expansion, ultimately resulting in clonal occupation of whole glands and supporting the "stem cell-hit" theory of endometrial carcinogenesis. Indeed, phosphatase and tensin homolog deleted from chromosome 10 (PTEN)null glands (due to a mutation and/or deletion in the PTEN gene), which are rarely observed in the normal endometrium, exhibit complete absence of PTEN expression in all cells that comprise the null gland [58]. These findings support the idea that the gland was occupied by descendants of a stem cell that had suffered a genetic "hit." A similar concept has been proposed regarding stem cells and the formation of crypts of the colon [59•].

Whether $\mathrm{CD} 133^{+} \mathrm{CSC}$ are derived from $\mathrm{CD} 133^{+}$cells in the normal endometrium is unknown. Similarly, although SP cells are observed in both normal and cancerous endometria, the relationship between them is unknown. Comparing the cancer predisposition between both fractions is interesting. We have established an in vitro model of endometrial carcinogenesis in which normal endometrial cells can be transformed by introducing defined genetic elements [60], leading to development of cancerous phenotypes [61]. Employing this model to compare the transforming efficiency of normal $\mathrm{CD} 133^{+} / \mathrm{SP}$ cells versus $\mathrm{CD} 133^{-} /$non-SP cells by introducing oncogenic elements may provide evidence supporting the potential origin of CSCs.

Additionally, some interesting concepts on the origin of CSCs have recently emerged. In hematological malignancies, the cells of origin have often been shown to be multipotential hematopoietic stem cells. On the other hand, a theory has been proposed that some leukemias also may develop from more committed progenitors that have reacquired stem cell properties. This idea is supported by the finding that transfection with oncogenic fusion genes, such as monocytic leukemia zinc finger protein-transcriptional intermediary factor 2 fusions, can confer granulocytemonocyte progenitors (GMPs) with leukemic properties [62]. As for chronic myeloid leukemia (CML) patients in blast crisis, CML GMPs are known to obtain self-renewing CSC phenotypes, unlike normal GMPs [63]. Activation of $\beta$-catenin in CML GMPs appears to enhance the selfrenewal activity and leukemic potential of these cells. Thus, more committed progenitors or de-differentiation of cells that have already gone through some stage of differentiation may be the origin of CSCs. Furthermore, the possibility that fully differentiated, yet proliferatively competent cells also can undergo malignant transformation cannot be excluded.

\section{Molecular Manipulation to Target CSCs for Cancer Therapy}

Although the percentage of CSCs in tumors varies among patients, CSCs are usually thought to be rare, and most of the cells occupying tumors are non-CSCs. Conventional chemotherapy targets the latter population; CSCs can survive these treatments, expand, and generate relapsed foci. Eradication of CSCs by molecular targeting leads to disabling of the self-renewal capacity, theoretically inhibiting regrowth of daughter cells.

What kinds of strategies or agents can be considered to target CSCs? Targeting key signaling pathways that are active in CSC self-renewal is one approach to therapy (Fig. 1). The representative signaling pathways critical for CSC self-renewal are the Wnt, hedgehog, and Notch pathways. A number of techniques have been reported to target these pathways [64]. The Notch pathway has traditionally been targeted with inhibitors of the protease $\gamma$-secretase, which inhibits the release of Notch intracellular domain. Combined exposure to the $\gamma$ secretase inhibitor ( $\mathrm{N}$-[N-(3,5-difluorophenacetyl)-L-alanyl]-S-phenylglycine t-butyl ester (DAPT)) and irradiation decreases the self-renewal capacity of human glioblastoma cells in association with a significant reduction in $\mathrm{CD}_{133^{+}}$cells, whereas irradiation alone has little effect [65]. Treatment of human colorectal cancer xenografts with the anti-human Notch ligand, Delta-like 4, 


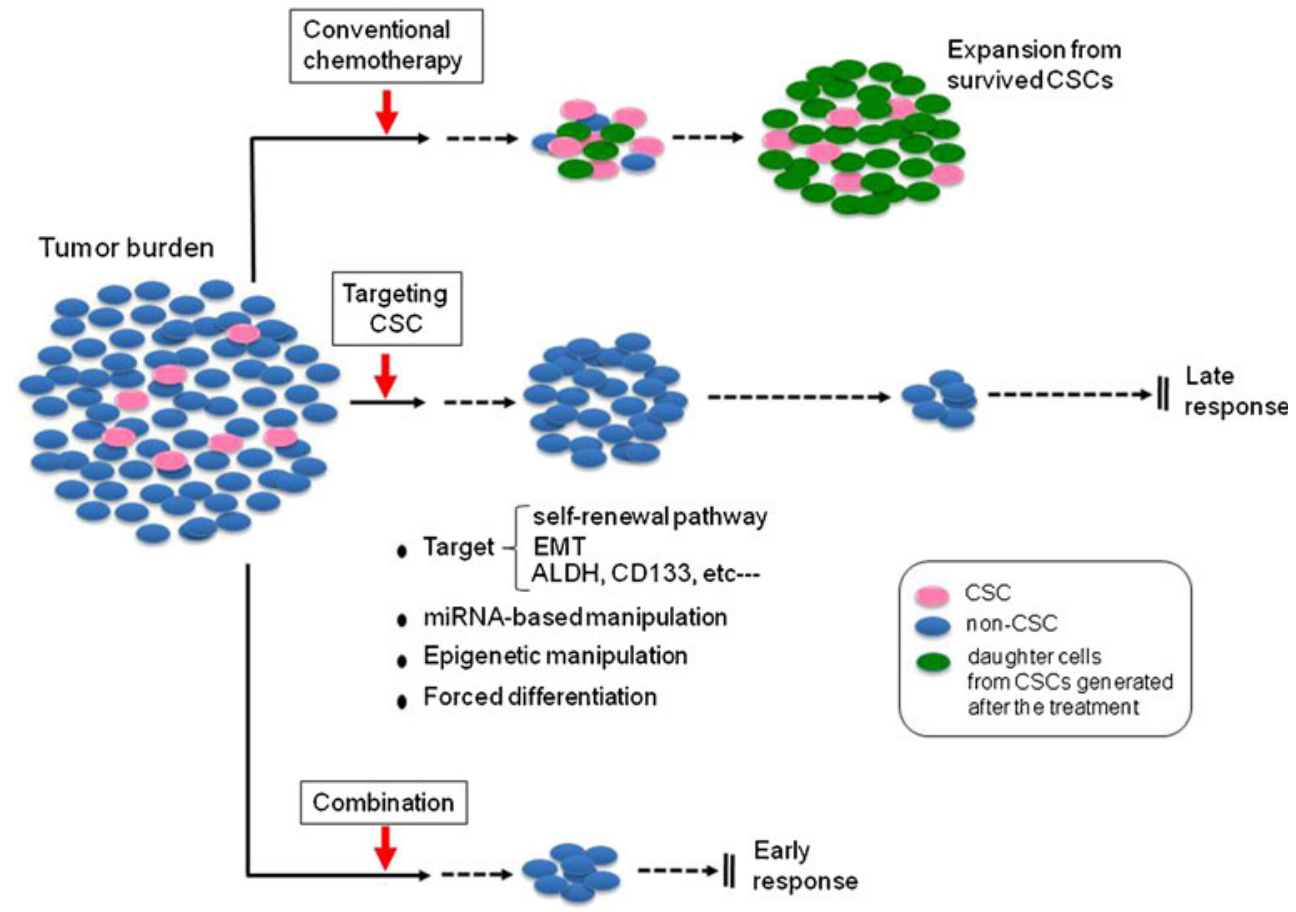

Fig. 1 The concept of targeting cancer stem cells (CSCs) combined with conventional chemotherapy. Conventional chemotherapy can act on non-cancer stem cells (non-CSCs) that occupy most of the tumor and reduce tumor volume in a relatively short period, but CSCs can survive such therapies, expand, and generate relapsed foci. Targeting the CSCs with molecular manipulations results in decreased numbers of CSCs, thereby inhibiting production of daughter cells. In this case, non-CSCs may have a limited potential for cell division, because some will undergo differentiation, senescence, apoptosis, or eradication by the immune system. As a result, tumor burden may be gradually reduced but will take a considerably longer period of time. A combination of both strategies can eradicate chemoresistant CSCs and reduce the tumor volume with a much earlier response, hopefully leading to complete response. EMT, epithelial-mesenchymal transition; ALDH, aldehyde dehydrogenase either alone or in combination with the chemotherapeutic agent irinotecan, reduces CSC frequency, whereas irinotecan treatment alone increases their frequency [66].

Epithelial-mesenchymal transition (EMT) is widely accepted to be tightly associated with the CSC phenotype [67]. Therefore, factors that regulate EMT may be alternative molecular targets in CSCs. Transforming growth factor (TFG) $\beta$ is a representative upstream inducer of EMT. The chemotherapeutic drug doxorubicin activates TGF- $\beta$ signaling, induces EMT, and promotes invasion, thus enhancing the generation of CSCs in murine breast cancer cells in vitro. A TGF- $\beta$ type I receptor kinase inhibitor (T $\beta$ RI-KI) inhibits doxorubicin-induced EMT and mammosphere formation, and a combination of doxorubicin and T $\beta$ RI-KI enhances the efficacy of doxorubicin in reducing tumor growth and lung metastasis in an orthotopic xenograft model compared to treatment with a single agent [68].

Cancers are widely known to have a specific microRNA (miR) expression profile in each tumor type [69] that differentially regulates the key properties of CSCs, including cellcycle exit and differentiation, prosurvival and antistress mechanisms, EMT, migration, and invasion, which all contribute to enhanced tumor initiation and metastatic potential
[70]. The miR-17-92 cluster has been reported as the first group of oncogenic miRNAs to accelerate tumor development in lung, breast, stomach, prostate, colon, and pancreatic cancers by regulating c-Myc expression [71]. The first evidence of tumor suppressive miRNAs was the miR-15/miR16 cluster, which often is deleted in chronic lymphocytic leukemias [72]. Dysregulated miRNAs are highly associated with tumor initiation, tumor maintenance, metastasis, and therapy resistance by modulating CSC properties. Studies have shown the potential implications of miRNA-based therapeutics as a novel strategy to target CSCs. To eliminate CSCs, oncogenic miRNAs can be targeted by locally administered antagomirs, and those recognized as tumor suppressors can be up-regulated using an appropriate viral vector system [73]. In glioblastomas, transfection of miR-124 and 137, which are down-regulated in this tumor type, causes cell cycle arrest and differentiation of CD133 ${ }^{+}$CSCs [74]. In medulloblastoma, introduction of miR-199b-5p inhibits the proliferation of engrafted CD133 ${ }^{+}$CSCs [75].

Epigenetic manipulations are proposed to reduce CSCs by acting on DNA acetylation and methylation. Histone acetylases (HDACs) are involved in the repression of various tumor suppressor genes, and HDAC inhibitors are used 
to induce re-expression of these genes. Kato et al. isolated SP and non-SP cells derived from a rat endometrial cell line expressing human [[12] Val] KRAS (RK12V cells) and determined the SP phenotype [76]. RK12V-SP cells showed self-renewal capacity, the potential to develop into stromal cells, reduced expression levels of differentiation markers, long-term proliferation capacity in culture, and enhanced tumorigenicity, indicating that RK12V-SP cells have CSClike features. Treatment with the HDAC inhibitor, sodium butyrate, reduced the self-renewal capacity and completely suppressed colony formation of RK12V-SP cells in soft agar. HDAC inhibitors may thus represent a promising type of antitumor therapy by targeting CSCs.

$\mathrm{ALDH}$, which is a member of a family of $\mathrm{NAD}(\mathrm{P})^{+}-$ dependent enzymes and a representative CSC marker in a variety of tumor types, also is an attractive therapeutic target. A number of strategies exist to eradicate cells with high levels of ALDH. Treatment of breast cancer cells with sulforaphane, a natural compound derived from broccoli/ broccoli sprouts, decreases the $\mathrm{ALDH}^{+}$cell population and the size and number of mammospheres, and abrogates tumor growth after reimplantation of primary tumor cells into secondary mice. This experiment demonstrates the elimination of CSCs and concomitant suppression of the $\mathrm{Wnt} / \beta$ catenin self-renewal pathway [77].

Forcing CSCs to differentiate by treating them with differentiation-inducing agents is an alternative way to target CSCs, based on the concept that inducing differentiation of CSCs may reduce their stem cell properties, leading to reduced tumor-forming ability. In glioblastomas, bone morphogenetic proteins (BMPs) activate their cognate receptors (BMPRs) and trigger the Smad signaling cascade. This is followed by reduced proliferation and increased neural differentiation, concomitant with reduced clonogenic ability, $\mathrm{CD}_{133^{+}}$population size, and xenograft tumor growth, indicating that BMP4 inhibits tumorigenicity by suppressing CSC activity via forced differentiation [78]. In breast cancer cells, treatment with all-trans retinoic acid reduces their sphereforming efficiency by promoting stem cell differentiation [79].

\section{Practical Strategies to Target Endometrial CSCs}

An amazingly diverse range of approaches to eradicate stem cells in cancer is becoming apparent. However, information on CSCs in endometrial cancer is sparse compared to other tumor types. CD133 expression and/or SP cells represent CSC characteristics in endometrial cancer $[31 \bullet, 32 \bullet, 33 \bullet]$. We have analyzed the relationship between $\mathrm{CD} 133^{+}$and SP cells, but these populations of cells did not stringently overlap (unpublished data). This finding raises several possibilities: Do overlapping populations identify legitimate CSCs? Alternatively, $\mathrm{CD} 133^{+}$cells and SP cells are independent populations, both of which have CSC phenotypes. Furthermore, additional populations with CSC phenotypes and distinct marker expression profiles are possible. If the overlapping population includes legitimate CSCs, simply targeting either $\mathrm{CD} 133^{+}$cells or SP cells will theoretically be effective in eradicating CSCs. In contrast, if $\mathrm{CD}_{133^{+}}$and SP fractions are independent CSC populations, both fractions should be simultaneously targeted. Fortunately, as shown above, several techniques to molecularly target cells with these markers have been reported, such as the $\gamma$-secretase inhibitor DAPT or miR-199b-5p, both of which inhibit the Notch pathway in $\mathrm{CD} 133^{+}$cells, and sodium butyrate, which targets SP cells.

Theoretically, targeting CSCs will lead to inhibition of the self-renewal capacity of CSCs, thereby ceasing generation of new daughter cells. In such a case, what is the fate of non-CSCs that mainly occupy the tumor? Non-CSCs are expected to have a limited potential for cell division, and some may undergo differentiation, senescence, apoptosis, or eradication by the immune system. As a result, tumor burden may be reduced, but it will take a considerably longer period of time. Therefore, combining the use of conventional chemotherapeutic agents with molecular targeting of CSCs is expected to decrease tumor burden more quickly, leading to an earlier clinical response (Fig. 1). Recently, a novel hypothesis was proposed stating that differentiated non-CSC cells can reacquire CSC features via dedifferentiation due to signals from the microenvironment and stromal cells [80]. Eradication of non-CSCs appears to be important for blocking this process, further supporting the role of conventional chemotherapy.

The challenge of finding additional CSC markers in endometrial cancer is an attractive task for identifying alternative targets. We have identified CD117 as another candidate CSC marker (manuscript in preparation). We examined this marker because a well-known molecular targeting agent, Imatinib, specifically targets CD117. We are currently using this agent combined with conventional chemotherapy and have determined that Imatinib effectively inhibits tumor growth in vivo in combination with cisplatin and have found that Imatinib sensitizes endometrial cancer cells to cisplatin. Regarding endometrial cancer, the taxan/platinum combination is now widely used for both adjuvant and cytoreductive settings. Combining taxan/platinum with CSC targeting agents is thus practical. Once CSC targeting agents are identified and validated, we should test their combinatorial effect with taxane/ platinum preclinically. In any case, specific targeting may prevent unwanted toxicity.

\section{Conclusion and Future Direction}

Although information on CSCs in endometrial cancer is sparser than that for other tumor types, CD133 expression 
and/or the phenotypes of SP cells are characteristics of CSCs. Additional markers may be discovered in the future, and we should resolve the question of the best marker for legitimate CSCs or whether multiple types of CSCs are present that express different markers. Once critical markers are identified, we should attempt to target CSCs, using on the accumulating molecular techniques.

One promising idea is to combine conventional chemotherapy with targeting CSCs, based on the concept that CSCs are chemoresistant and may survive conventional chemotherapy, whereas non-CSCs that occupy most of the tumor may not be effectively killed by CSC targeting. Pharmacological interaction between CSC targeting agents and taxan/platinum should be extensively investigated to find the best combination to achieve the maximal additive or hopefully synergistic effects. In any case, specific targeting may prevent unwanted toxicity.

Importantly, more profound understanding of CSCs can provide greater insight into the biology and mechanisms of carcinogenesis in endometrial cancer. This may be a promising shortcut to success in developing novel therapeutic modalities for this tumor type.

Acknowledgments The author thanks all of the members of the molecular pathology group of his department for their tremendous contribution to the experiments involving stem cells in the human endometrium as well as endometrial cancer: Drs. Yoshiko Maida, Masahiro Takakura, Yasunari Mizumoto, Mitsuhiro Nakamura, Noriko Mori, Yukiko Bono, Xiuzhi Zhang. The studies were supported by a Grant-in-Aid for Scientific Research from the Japan Society for the Promotion of Science (JSPS) and the Megumi Medical Foundation of Kanazawa University.

Disclosure No potential conflicts of interest relevant to this article were reported.

\section{References}

Papers of particular interest, published recently, have been highlighted as:

- Of importance

1. Prianishnikov VA. On the concept of stem cell and a model of functional-morphological structure of the endometrium. Contraception. 1978;18:213-23.

2. Padykula HA, Coles LG, McCracken JA, King Jr NW, Longcope C, Kaiserman-Abramof IR. A zonal pattern of cell proliferation and differentiation in the rhesus endometrium during the estrogen surge. Biol Reprod. 1984;31:1103-18.

3. Padykula HA. Regeneration in the primate uterus: the role of stem cells. Ann N Y Acad Sci. 1991;622:47-56.

4. Kyo S, Maida Y, Inoue M. Stem cells in endometrium and endometrial cancer: accumulating evidence and unresolved questions. Cancer Lett. 2011;308:123-33.

5. Tanaka M, Kyo S, Kanaya T, Yatabe N, Nakamura M, Maida Y, et al. Evidence of monoclonal composition of human endometrial epithelial glands and mosaic pattern of clonal distribution in luminal epithelium. Am J Pathol. 2003;163:295-301.

6. Okabe M, Ikawa M, Kominami K, Nakanishi T, Nishimune Y. 'Green mice' as a source of ubiquitous green cells. FEBS Lett. 1997;407:313-9.

7. Hadjantonakis AK, Cox LL, Tam PP, Nagy A. An X-linked GFP transgene reveals unexpected paternal X-chromosome activity in trophoblastic giant cells of the mouse placenta. Genesis. 2001;29:133-40.

8. Chan RW, Schwab KE, Gargett CE. Clonogenicity of human endometrial epithelial and stromal cells. Biol Reprod. 2004;70:1738-50.

9. Goodell MA, Brose K, Paradis G, Conner AS, Mulligan RC. Isolation and functional properties of murine hematopoietic stem cells that are replicating in vivo. J Exp Med. 1996;183:1797-806.

10. Zhou S, Schuetz JD, Bunting KD, Colapietro AM, Sampath J, Morris JJ, et al. The ABC transporter Bcrp1/ABCG2 is expressed in a wide variety of stem cells and is a molecular determinant of the side-population phenotype. Nat Med. 2001;7:1028-34.

11. Kato K, Yoshimoto M, Kato K, Adachi S, Yamayoshi A, Arima T, et al. Characterization of side-population cells in human normal endometrium. Hum Reprod. 2007;22:1214-23.

12. Gargett CE, Schwab KE, Zillwood RM, Nguyen HP, Wu D. Isolation and culture of epithelial progenitors and mesenchymal stem cells from human endometrium. Biol Reprod. 2009;80:1136-45.

13. Masuda H, Matsuzaki Y, Hiratsu E, Ono M, Nagashima T, Kajitani T, et al. Stem cell-like properties of the endometrial side population: implication in endometrial regeneration. PLoS One. 2010;5:e10387.

14. Cervelló I, Gil-Sanchis C, Mas A, Delgado-Rosas F, MartínezConejero JA, Galán A, Martínez-Romero A, Martínez S, Navarro I, Ferro J, Horcajadas JA, Esteban FJ, O'Connor JE, Pellicer A, Simón C. Human endometrial side population cells exhibit genotypic, phenotypic and functional features of somatic stem cells. PLoS One. 2010;5:e10964.

15. Visvader JE, Lindeman GJ. Cancer stem cells in solid tumours: accumulating evidence and unresolved questions. Nat Rev Cancer. 2008;8:755-68.

16. Lapidot T, Sirard C, Vormoor J, Cashman JD, Doedens M, Murdoch $\mathrm{B}$, et al. A cell initiating human acute myeloid leukaemia after transplantation into SCID mice. Nature. 1994;367:645-8.

17. Bonnet D, Dick JE. Human acute myeloid leukemia is organized as a hierarchy that originates from a primitive hematopoietic cell. Nat Med. 1997;3:730-7.

18. Bao S, et al. Glioma stem cells promote radioresistance by preferential activation of the DNA damage response. Nature. 2006;444:756-60.

19. Prince ME, et al. Identification of a subpopulation of cells with cancer stem cell properties in head and neck squamous cell carcinoma. Proc Natl Acad Sci USA. 2007;104:973-8.

20. Ginestier C, et al. ALDH1 is a marker of normal and malignant human mammary stem cells and a predictor of poor clinical outcome. Cell Stem Cell. 2007;1:555-67.

21. Al-Hajj M, et al. Prospective identification of tumorigenic breast cancer cells. Proc Natl Acad Sci USA. 2003;100:3983-8.

22. Eramo A, et al. Identification and expansion of the tumorigenic lung cancer stem cell population. Cell Death Differ. 2008;15:504-14.

23. Jiang F, et al. Aldehyde dehydrogenase 1 is a tumor stem cellassociated marker in lung cancer. Mol Cancer Res. 2009;7:330-8.

24. Ding W, et al. CD133+ liver cancer stem cells from methionine adenosyl transferase $1 \mathrm{~A}$-deficient mice demonstrate resistance to transforming growth factor (TGF)-beta-induced apoptosis. Hepatology. 2009;49:1277-86.

25. Yang ZF, et al. Significance of $\mathrm{CD} 90^{+}$cancer stem cells in human liver cancer. Cancer Cell. 2008;13:153-66.

26. Takaishi $\mathrm{S}$, et al. Identification of gastric cancer stem cells using the cell surface marker CD44. Stem Cells. 2009;27:1006-20.

27. Li C, et al. Identification of pancreatic cancer stem cells. Cancer Res. 2007;67:1030-7. 
28. Hermann PC, et al. Distinct populations of cancer stem cells determine tumor growth and metastatic activity in human pancreatic cancer. Cell Stem Cell. 2007;1:313-23.

29. O'Brien CA, et al. A human colon cancer cell capable of initiating tumour growth in immunodeficient mice. Nature. 2007;445:106-10.

30. Ricci-Vitiani L, et al. Identification and expansion of human coloncancer-initiating cells. Nature. 2007;445:111-5.

31. - Nakamura M, Kyo S, Zhang B, Zhnag X, Mizumoto Y, Takakura M, et al. Prognostic impact of CD133 expression as a tumor initiating marker in endometrial cancer. Hum Pathol. 2010;41:1516-29. This study revealed that $C D 133^{+}$endometrial cancer stem cells contribute to worse prognosis of the patients. High expression of CD133 in tumor was an independent prognostic factor in this tumor type.

32. - Rutella S, Bonanno G, Procoli A, Mariotti A, Corallo M, Prisco MG, et al. Cells with characteristics of cancer stem/progenitor cells express the CD133 antigen in human endometrial tumors. Clin Cancer Res. 2009;15:4299-311. This study for the first time identified CD133 cells with properties of cancer stem cells in endometrial cancer.

33. - Kato K, Takao T, Kuboyama A, Tanaka Y, Ohgami T, Yamaguchi S, et al. Endometrial cancer side-population cells show prominent migration and have a potential to differentiate into the mesenchymal cell lineage. Am J Pathol. 2010;176:381-92. This study identified sidepopulation cells in endometrial cancer with characterestics of cancer stem cell. Interesting results are that they can differentiate into mesencymal cells.

34. Stewart JM, et al. Phenotypic heterogeneity and instability of human ovarian tumor-initiating cells. Proc Natl Acad Sci USA. 2011;108(16):6468-73.

35. Silva IA, et al. Aldehyde dehydrogenase in combination with CD133 defines angiogenic ovarian cancer stem cells that portend poor patient survival. Cancer Res. 2011;71:3991-4001.

36. Vander Griend DJ, et al. The role of CD133 in normal human prostate stem cells and malignant cancer-initiating cells. Cancer Res. 2008;68:9703-11.

37. Collins AT, et al. Prospective identification of tumorigenic prostate cancer stem cells. Cancer Res. 2005;65:10946-51.

38. Jaksch M, et al. Cell cycle-dependent variation of a CD133 epitope in human embryonic stem cell, colon cancer, and melanoma cell lines. Cancer Res. 2008;68:7882-6.

39. Schatton $T$, et al. Identification of cells initiating human melanomas. Nature. 2008;451:345-9.

40. Hubbard SA, Friel AM, Kumar B, Zhang L, Rueda BR, Gargett CE. Evidence for cancer stem cells in human endometrial carcinoma. Cancer Res. 2009;69:8241-8.

41. Cheng JX, Liu BL, Zhang X. How powerful is CD133 as a cancer stem cell marker in brain tumors? Cancer Treat Rev. 2009;35:403-8.

42. Zhang M, Song T, Yang L, Chen R, Wu L, Yang Z, Fang J. Nestin and CD133: valuable stem cell-specific markers for determining clinical outcome of glioma patients. J Exp Clin Cancer Res. 2008;27:85.

43. Horst D, Scheel SK, Liebmann S, Neumann J, Maatz S, Kirchner T, Jung A. The cancer stem cell marker CD133 has high prognostic impact but unknown functional relevance for the metastasis of human colon cancer. J Pathol. 2009;219:427-34.

44. Maeda S, Shinchi H, Kurahara H, Mataki Y, Maemura K, Sato M, Natsugoe S, Aikou T, Takao S. CD133 expression is correlated with lymph node metastasis and vascular endothelial growth factor-C expression in pancreatic cancer. Br J Cancer. 2008;98:1389-97.

45. Chen YC, et al. Aldehyde dehydrogenase 1 is a putative marker for cancer stem cells in head and neck squamous cancer. Biochem Biophys Res Commun. 2009;385:307-13.

46. Rasheed ZA, et al. Prognostic significance of tumorigenic cells with mesenchymal features in pancreatic adenocarcinoma. J Natl Cancer Inst. 2010;102:340-51.

47. $\mathrm{Li} \mathrm{T}$, et al. ALDH1A1 is a marker for malignant prostate stem cells and predictor of prostate cancer patients' outcome. Lab Invest. 2010;90:234-44.
48. Ran D, et al. Aldehyde dehydrogenase activity among primary leukemia cells is associated with stem cell features and correlates with adverse clinical outcomes. Exp Hematol. 2009;37:1423-34.

49. Cheung AM, et al. Aldehyde dehydrogenase activity in leukemic blasts defines a subgroup of acute myeloid leukemia with adverse prognosis and superior NOD/SCID engrafting potential. Leukemia. 2007;21:1423-30.

50. Charafe-Jauffret E, et al. Aldehyde dehydrogenase 1-positive cancer stem cells mediate metastasis and poor clinical outcome in inflammatory breast cancer. Clin Cancer Res. 2010;16:45-55.

51. Tanei $\mathrm{T}$, et al. Association of breast cancer stem cells identified by aldehyde dehydrogenase 1 expression with resistance to sequential Paclitaxel and epirubicin-based chemotherapy for breast cancers. Clin Cancer Res. 2009;15:4234-41.

52. Morimoto K, et al. Stem cell marker aldehyde dehydrogenase 1positive breast cancers are characterized by negative estrogen receptor, positive human epidermal growth factor receptor type 2, and high Ki67 expression. Cancer Sci. 2009;100:1062-8.

53. Gelmini S, et al. The critical role of SDF-1/CXCR4 axis in cancer and cancer stem cells metastasis. J Endocrinol Invest. 2008;31:809-19.

54. Gelmini S, et al. The CXCR4/CXCL12 axis in endometrial cancer. Clin Exp Metastasis. 2009;26:261-8.

55. Krohn A, et al. CXCR4 receptor positive spheroid forming cells are responsible for tumor invasion in vitro. Cancer Lett. 2009;280:65-71.

56. Charafe-Jauffret E, et al. Breast cancer cell lines contain functional cancer stem cells with metastatic capacity and a distinct molecular signature. Cancer Res. 2009;69:1302-13.

57. Phesse TJ, Clarke AR. Normal stem cells in cancer prone epithelial tissues. Br J Cancer. 2009;100:221-7.

58. Mutter GL, Lin MC, Fitzgerald JT, Kum JB, Baak JP, Lees JA, et al. Altered PTEN expression as a diagnostic marker for the earliest endometrial precancers. J Natl Cancer Inst. 2000;92:924-30.

59. Baker N, et al. Crypt stem cells as the cells-of-origin of intestinal cancer. Nature. 2009;457:608-11. This study clearly shows that normal stem cells as the cell of origin for neoplasia and that transformation of the intestinal stem cells is a primary route to intestinal neoplasia.

60. Kyo, et al. Successful immortalization of endometrial glandular cells with normal structural and functional characteristics. Am J Pathol. 2003;163:2259-69.

61. Mizumoto $\mathrm{Y}$, et al. Creation of tumorigenic human endometrial epithelial cells with intact chromosomes by introducing defined genetic elements. Oncogene. 2006;25:5673-82.

62. Chan WI, Huntly BJ. Leukemia stem cells in acute myeloid leukemia. Semin Oncol. 2008;35:326-35.

63. Jamieson, et al. Glanulocyte-macrophage progenitors as candidate leukemic stem cells in blast-crisis CML. N Engl J Med. 2004;351:657-67.

64. Alison MR, Lim SM, Nicholson LJ. Cancer stem cells: problems for therapy? J Pathol. 2011;223:147-61.

65. Hovinga, et al. Inhibition of notch signaling in glioblastoma targets cancer stem cells via an endothelial cell intermediate. Stem Cells. 2010;28:1019-29.

66. Hoey, et al. DLL4 blockade inhibits tumor growth and reduces tumor-initiating cell frequency. Cell Stem Cell. 2009;5:168-77.

67. Thiery JP, et al. Epithelial-mesenchymal transitions in development and disease. Cell. 2009;139:871-90.

68. Bandyopadhyay A, et al. Doxorubicin in combination with a small TGFbeta inhibitor: a potential novel therapy for metastatic breast cancer in mouse models. PLoS One. 2010;5:e10365.

69. Nicoloso MS, et al. MircoRNAs-the micro steering wheel of tumor metastases. Nat Rev Cancer. 2009;9:293-302.

70. Garg M. MicroRNAs, stem cells and cancer stem cells. World J Stem Cells. 2012;4:62-70. 
71. He L, et al. A microRNA polycistron as a potential human oncogene. c-Myc-regulated microRNAs modulate E2F1 expression. Nature. 2005;435:828-33.

72. Calin GA, et al. Frequent deletions and down-regulation of microRNA genes miR15 and miR16 at 13q14 in chronic lymphocytic leukemia. Proc Natl Acad Sci USA. 2002;99:15524-9.

73. Calin GA, Croce CM. MicroRNA signatures in human cancers. Nat Rev Cancer. 2006;6:857-66.

74. Silber J, et al. miR-124 and miR-137 inhibit proliferation of glioblastoma multiforme cells and induce differentiation of brain tumor stem cells. BMC Med. 2008;6:14.

75. Garzia L, et al. MicroRNA-199b-5p impairs cancer stem cells through negative regulation of HSE in medulloblastoma. ProS One. 2009;4:e4998.
76. Kato K, et al. Sodium butyrate inhibits the self-renewal capacity of endometrial tumor side-population cells by inducing a DNA damage response. Mol Cancer Ther. 2011;10:1430-9.

77. Li Y, et al. Sulforaphane, a dietary component of broccoli/broccoli sprouts, inhibits breast cancer stem cells. Clin Cancer Res. 2010;16:2580-90.

78. Piccirillo SG, et al. Bone morphogenetic proteins inhibit the tumorigenic potential of human brain tumour-initiating cells. Nature. 2006;444:761-5.

79. Ginestier $C$, et al. Retinoid signaling regulates breast cancer stem cell differentiation. Cell Cycle. 2009;8:3297-302.

80. Vermeulen L, et al. The developing cancer stem-cell model: clinical challenges and opportunities. Lancet Oncol. 2012;13: e83-9. 